Proc Indian Natn Sci Acad 81 No. 1 February Special Issue 2015 pp. 267-271

(C) Printed in India. $\quad$ DOI: $10.16943 /$ ptinsa/2015/v81i1/48080

\title{
Development of Secular Instability in Different Disc Models of Black Hole Accretion
}

\author{
SANKHASUBHRA NAG ${ }^{* 1}$, DEEPIKA B ANANDA ${ }^{2}$, ISHITA MAITY ${ }^{3}$ and TAPAS K. DAS ${ }^{4}$ \\ ${ }^{1}$ Department of Physics, Sarojini Naidu College for Women, Kolkata 700 028, India \\ ${ }^{2}$ Indian Institute of Science Education and Research, Pune 411 008, India \\ ${ }^{3}$ Louisiana State University, LA 70803-4001, USA \\ ${ }^{4}$ Harish-Chandra Research Institute, Allahabad 211 019, India
}

(Received on 01 May 2014; Accepted on 27 August 2014)

\begin{abstract}
Analytical treatment of black hole accretion generally presumes the stability of the stationary configuration. Various authors in past decade actually demonstrated the validity of such an assumption at least for inviscid hydrodynamic flow. Inviscid condition is a reasonable approximation for low angular momentum advection dominated flow in connection to certain supermassive black holes at the centres of the galaxies (including our own) fed from a number of stelar donors. Introduction of a weak viscosity, however, may sometimes provide a more detail understanding of the observed spectrum. But recently, some of the authors demonstrated that an introduction of slightest amount of viscosity in quasi-viscous form makes a stationary accretion disc (in vertical equilibrium model) unstable. We made the same sort of analysis in other disc models for quasi-viscous models under post-Newtonian scheme. We introduced perturbations on the stationary flow solution particularly in standing wave form and studied their time evolution to observe whether they grow with time. Our analysis shows that same sort of secular instability exists in other disc models too. We further argued that with sufficiently low value of viscosity in the realistic Astrophysical perspective the instability does not effectively jeopardise the stationary condition.
\end{abstract}

\section{Key Words : Black Hole; Accretion; Disc Model}

\section{Introduction}

Astrophysical accretion is an important phenomena in connection to the observational identifications of black hole candidates (Frank et al., 2002). As the general MHD solution in this case is almost impossible to obtain analytically, people generally use hydrodynamical models to get an approximate analytical solution (see Chaudhury et al., 2006; Nag et al., 2012 and references therein). But in analytical analysis, generally

*Author for Correspondence : E-mail: sankhasubhra_nag@yahoo.co.in 
the features of the stationary configuration are studied under different conditions such as equation of state, disc geometries, dynamical formalisms etc. But all those analysis implicitly presume the stability of the stationary conditions. But recently some of the authors (e.g. Bhattacharjee and Ray, 2007; Bhattacharjee et al., 2009) demonstrated that with the introduction of a very small amount of viscosity may give rise to instabilities. But all the results available in the literature were worked out in a particular type of disc model, i.e. the model based on hydrostatic equilibrium along vertical equilibrium (henceforth to be referred as VE) and with adiabatic equation of state only. We generalised these studies to all the disc models available in the literature (see Chakrabarti and Das, 2001).

\section{Basic Equations}

If some fluid is being accreted forming a disc like structure into some accretor, the rate of incoming mass into a thin ring shaped region of radius $r$ must be equal to the rate of increase of fluid mass in the region. Denoting the local surface mass density on the disc by $\Sigma$ and the inward radial velocity of the fluid by $v$, this consideration gives the continuity equation (Frank et al., 2002),

$$
\frac{\partial}{\partial t}(\Sigma)+\frac{1}{r} \frac{\partial}{\partial r}(\Sigma v r)=0
$$

where $\Sigma=\rho H, \rho$ being the local density and $H$ being local disc half width.

The dependence of $H$ on radial distance $r$ varies in different disc models available (Chakrabarti and Das, 2001) in the literature. These are,

$$
\begin{aligned}
& H=H_{0} \quad \text { for constant height disc }(\mathrm{CH}) \text {, } \\
& H=\Theta r, \quad \Theta \text { being a constant } \quad \text { for conical flow model (CM), } \\
& H=c_{s}(r) \sqrt{\frac{r}{\gamma \phi^{\prime}(r)}} \quad \text { for vertical equilibrium model (VE), }
\end{aligned}
$$

where, $c_{s}(r)=\sqrt{\partial P / \partial \rho}$ is the local acoustic speed with polytropic equation of state $P=K \rho^{\gamma}$ ( $P$ being local pressure and $P=K \rho$ in isothermal cases).

Now for all the models $\rho H$ may be written in the form $\rho^{1+\varepsilon} \bar{g}(r)$ for some constant $\epsilon$ and some function $\bar{g}(r)$, both of which vary from model to model.

For the disc geometry there must be azimuthal symmetry. But setting the Euler equation in lateral direction one gets the angular momentum Balance equation

$$
\rho H \frac{\partial}{\partial t}\left(r^{2} \Omega\right)+\rho v H \frac{\partial}{\partial r}\left(r^{2} \Omega\right)=\frac{1}{2 \pi r}\left(\frac{\partial G}{\partial r}\right)
$$


where $G=2 \pi \nu \Sigma r^{3} \frac{\partial \Omega}{\partial r}, \nu=\alpha c_{s} H$.

Euler equation in radial equation takes the form,

$$
\frac{\partial v}{\partial t}+v \frac{\partial v}{\partial t}+\frac{1}{\rho} \frac{\partial P}{\partial r}+\Phi^{\prime}(r)-\frac{\lambda_{0}^{2}}{r^{3}}-\frac{2 \alpha \lambda_{0} F}{r^{3}}=0
$$

where,

$$
r^{2} \Omega=\lambda_{0}+\alpha \lambda_{0} F\left(c_{s}, v, r\right) .
$$

Now, Eq. (1) may be recast as,

$$
\frac{\partial}{\partial t}\left(g_{1}(\rho)\right)+g_{2}(r) \frac{\partial}{\partial r}(f)=0
$$

where $g_{1}(\rho)=\rho^{1+\varepsilon}, g_{2}(\rho)=\frac{1}{r \bar{g}(r)}$ and $f(\rho, v, r)=\rho v r H$ and Eq. (3) may be written as (where $f_{1}=$ $\left.\rho c_{s} H^{2} r^{3}\right)$,

$$
\frac{1}{v} \frac{\partial}{\partial t}\left(r^{2} \Omega\right)+\frac{\partial}{\partial r}\left(r^{2} \Omega\right)=\frac{\alpha}{f} \frac{\partial}{\partial r}\left(f_{1} \frac{\partial \Omega}{\partial r}\right) .
$$

Now putting Eq. (5) in Eq. (7) one gets,

$$
F\left(c_{s}, v, r\right)=-2\left[\frac{f_{1}}{f r^{3}}+\int \frac{f_{1}}{f r^{3}}\left(\frac{1}{f} \frac{\partial f}{\partial r}\right) d r\right] .
$$

\section{Stationary Solutions}

Stationary flow means for all the flow variables, $\frac{\partial}{\partial t} \equiv 0$ Hence Eqs. (6) and (4) take the form

$$
\begin{aligned}
\frac{\partial f}{\partial r} & =0, \\
v \frac{\partial v}{\partial r}+\frac{1}{\rho} \frac{\partial P}{\partial r}+\Phi^{\prime}(r)-\frac{\lambda_{0}^{2}}{r^{3}}-\frac{2 \alpha \lambda_{0} F}{r^{3}} & =0 .
\end{aligned}
$$

\section{Introduction of Time Dependent Perturbation}

Now let us introduce a small time dependent perturbation on the stationary solution of the flow variables (denoted by suffix 0) at each radial distance $r$, such that,

$$
\begin{array}{r}
v(r, t)=v_{0}(r)+\tilde{v}(r, t), \\
\rho(r, t)=\rho_{0}(r)+\tilde{\rho}(r, t), \\
f(r, t)=f_{0}(r)+\tilde{f}(r, t), \\
g_{1}(r, t)=g_{10}(r)+\tilde{g_{1}}(r, t), \\
g_{2}(r, t)=g_{20}(r)+\tilde{g_{2}}(r, t), \\
F(r, t)=F_{0}(r)+\tilde{F}(r, t) .
\end{array}
$$


To investigate the stability of the stationary solution, one has to find whether these perturbing terms grow with time or not. Substituting these expressions into the Eq. (6) and (4) one gets an inhomogeneous wave equation,

$$
\frac{\partial}{\partial t}\left[\frac{v_{0}}{f_{0}} \frac{\partial \tilde{f}}{\partial t}\right]+\frac{\partial}{\partial t}\left[\frac{v_{0}^{2}}{f_{0}} \frac{\partial \tilde{f}}{\partial r}\right]+\frac{\partial}{\partial r}\left[\frac{v_{0}^{2}}{f_{0}} \frac{\partial \tilde{f}}{\partial t}\right]+\frac{\partial}{\partial r}\left[\frac{v_{0}}{f_{0}}\left(v_{0}^{2}-\frac{c_{s 0}^{2}}{1+\varepsilon}\right) \frac{\partial \tilde{f}}{\partial r}\right]-\frac{2 \alpha \lambda_{0}^{2}}{r^{3}} \frac{\partial \tilde{F}}{\partial t}=0
$$

where,

$$
\frac{\partial \tilde{F}}{\partial t}=\frac{c_{s 0}}{\rho_{0} v_{0} r^{2}} \frac{(1+\gamma+4 \varepsilon)}{(1+\varepsilon)} \frac{\partial \tilde{f}}{\partial r}+2 \int \frac{\partial}{\partial r}\left(\frac{c_{s 0}}{\rho_{0} v_{0}^{2} r^{2}}\right) \frac{\partial \tilde{f}}{\partial t} d r
$$

Now choosing a trial wave solution,

$$
\tilde{f}(r, t)=g_{\omega}(r) e^{-i \omega t}
$$

one gets may explore the behaviour of the spatial part. Hence substituting the above in Eq. (12), the equation becomes,

$$
\begin{aligned}
& \omega^{2} v_{0} g_{\omega}^{2}+v_{0}^{2} i \omega \frac{d g_{\omega}}{d r} g_{\omega}+i \omega \frac{d}{d r}\left(v_{0}^{2} g_{\omega}\right) g_{\omega}-g_{\omega} \frac{d}{d r}\left(v_{0}\left(v_{0}^{2}-\frac{c_{s 0}^{2}}{1+\varepsilon}\right) \frac{d g_{\omega}}{d r}\right)+ \\
& \quad \frac{2 \alpha \lambda_{0}^{2} c_{s 0} f_{0} g_{w}}{\rho_{0} v_{0} r^{5}}\left(\frac{1+\gamma+4 \varepsilon}{1+\varepsilon}\right) \frac{d g_{\omega}}{d r}-\frac{4 \alpha \lambda_{0}^{2} i \omega f_{0} g_{\omega}}{r^{3}} \int \frac{d}{d r}\left(\frac{c_{s 0}}{\rho_{0} v_{0}^{2} r^{2}}\right) g_{\omega} d r=0 .
\end{aligned}
$$

The solution of this equation may be of two types. One is of standing wave type, where $\tilde{f}$ takes the form $A_{\omega}(r, t) \exp (-i \Omega t)$ where both $A_{\omega}(r, t)$ and $\Omega$ are real. The other is of travelling wave type where $\tilde{f}$ takes the form $A_{\omega}(r, t) \exp [i(s(r)-\omega t)]$, all of $A_{\omega}(r, t), s(r)$ and $\omega$ being real. Here we will discuss the behaviour of the standing wave wave solutions and that of the travelling solutions will be reported elsewhere.

\section{Results and Comments}

Upon integrating the above equation so that the surface integral terms vanish we get finally something like $A \omega^{2}+B \omega+C=0$, where

$$
\begin{gathered}
A=\int v_{0} g_{\omega}^{2} d r, \quad B=-4 i \alpha \lambda_{0}^{2} \int \frac{g_{\omega} f_{0}}{r^{3}}\left[\int g_{\omega} \frac{d}{d r}\left(\frac{c_{s 0}}{\rho_{0} v_{0}^{2} r^{2}}\right) d r\right] d r \\
C=\int v_{0}\left(v_{0}^{2}-\frac{c_{s 0}^{2}}{1+\varepsilon}\right)\left(\frac{d g_{\omega}}{d r}\right)^{2}+2 \alpha \lambda_{0}^{2} \int \frac{c_{s 0} f_{0}}{\rho_{0} v_{0} r^{5}}\left(\frac{1+\gamma+4 \varepsilon}{1+\varepsilon}\right) g_{\omega} \frac{d g_{\omega}}{d r} d r \\
\operatorname{Re}(-i \omega)=\alpha\left[\int v_{0} g_{\omega}^{2} \xi(r) d r\right]\left[v_{0} g_{\omega}^{2} d r\right]^{-1} \sim \alpha \xi(r),
\end{gathered}
$$


where

$$
\xi(r)=\frac{2 \lambda_{0}^{2}}{r^{3} g_{w}} \frac{g_{10}}{g_{20}} \int g_{w} \frac{d}{d r}\left(\frac{c_{s 0} g_{10} \frac{1+2 \varepsilon}{1+\varepsilon}}{g_{20}^{2} f_{0}^{2} r^{2}}\right) d r .
$$

Now after putting the specific forms of $g_{1}, g_{2}$ and $\epsilon$, one gets for $\mathrm{CH}$ model, $\xi(r) \sim r^{-2}$ while for $\mathrm{CM}, \xi(r) \sim r^{1}$ and for $\mathrm{VE}, \xi(r) \sim r^{5 / 2}$; which readily implies that at large radial distance for CM \& $\mathrm{VE}$ the perturbation will rapidly grow with time making the disc unstable but for $\mathrm{CH}$ it will diminish with radial distance making the disc practically stable for large amount of time. Moreover in all cases with sufficiently smaller value of $\alpha$, the instability may grow too slowly to sustain the disc for large enough time in comparison with the lifespan of the disc otherwise realisable.

\section{References}

1. Bhattacharjee JK and Ray AK (2007) Secular instability in quasi-viscous disk accretion Astrophys $J 668409$

2. Bhattacharjee JK, Bhattacharya A, Das TK and Ray AK (2009) Quasi-viscous accretion flow - i. equilibrium conditions and asymptotic behaviour MNRAS 398841

3. Chakrabarti SK and Das S (2001) Model dependence of transonic properties of accretion flows around black holes MNRAS 327808

4. Chaudhury S, Ray AK and Das TK (2006) Critical properties and stability of stationary solutions in multitransonic pseudo-schwarzschild accretion MNRAS 373146

5. Frank J, King A and Raine D (2002) Accretion Power in Astrophysics Cambridge University Press

6. Nag S, Acharya S, Ray AK and Das TK (2012) The role of flow geometry in influencing the stability criteria for low angular momentum axisymmetric black hole accretion New Astronomy 17285. 